\title{
Revisiting Lévy flights on bounded domains: a Fock space approach
}

\author{
H A Araújo ${ }^{1,4}$, M O Lukin ${ }^{1}$, M G E da Luz ${ }^{2}$, \\ G M Viswanathan ${ }^{3}$, F A N Santos ${ }^{4,5}$ and \\ E P Raposo ${ }^{1,6,7}$
}

${ }^{1}$ Laboratório de Física Teórica e Computacional, Departamento de Física, Universidade Federal de Pernambuco, 50670-901, Recife-PE, Brazil

${ }^{2}$ Departamento de Física, Universidade Federal do Paraná, Curitiba-PR, 81531-980, Brazil

${ }^{3}$ Department of Physics and National Institute of Science and Technology of Complex Systems, Universidade Federal do Rio Grande do Norte, 59078-970, Natal-RN, Brazil

${ }^{4}$ Departamento de Matemática, Universidade Federal de Pernambuco, 50670-901, Recife-PE, Brazil

${ }^{5}$ Vrije Universiteit Amsterdam, Amsterdam Neuroscience, 1081 HZ, Amsterdam, The Netherlands

${ }^{6}$ Centre d'Estudis Avançats de Blanes-CEAB-CSIC, Girona, 17300, Spain, and Centre for Ecological Research and Forestry Applications-CREAF, Universitat Autònoma de Barcelona, 08193, Cerdanyola del Vallès, Spain E-mail: ernesto@df.ufpe.br

Received 27 March 2020

Accepted for publication 28 June 2020

Published 5 August 2020

Online at stacks.iop.org/JSTAT/2020/083202

https://doi.org/10.1088/1742-5468/aba593

\begin{abstract}
The statistical description of a one-dimensional superdiffusive Lévy flier restricted to a finite domain is well known to be technically involving. For example, in this type of process the probability distribution $P(x, t)$ and survival probability $S(t)$ cannot be obtained from the method of images. Other methods, such as the fractional derivative approach, also find technical difficulties due to the long jumps combined with the presence of absorbing boundaries. Here we revisit this problem through a different point of view. We map the corresponding master equation to a Schrödinger-like equation and then describe the Lévy flier evolution in a Fock space. The system states are assigned to the available
\end{abstract}

\footnotetext{
${ }^{7}$ Author to whom any correspondence should be addressed.
} 
positions in the discrete space. The Hamiltonian-like matrix is calculated for any Lévy index $\alpha \in(0,2]$. For the system sizes studied here the computation of its eigenvalues and eigenvectors are performed using a symbolic computing software. This method allows to build the time evolution operator, the distribution $P(x, t)$ and the survival probability $S(t)$. We compare our results for $P(x, t)$ with direct Monte Carlo simulations and find a good agreement for all $\alpha \in(0,2]$. Similarly, our results for $S(t)$ nicely agree with the numerical simulations for any time $t$, including the short-term behavior. In the long-term asymptotic limit we identify the crossover between the power-law and exponential behaviors, which emerge respectively when only one or both boundaries are reached by the Lévy flier. Comparisons with some exact expressions for Lévy flights in the continuous space limit also display good agreement. We conclude our analysis by discussing the possibility of extending the present framework to general bounded random walks and flights.

Keywords: stochastic particle dynamics, random searches, Lévy distribution, Fock space

\section{Contents}

1. Introduction

2. Fock space formalism for bounded random particles

3. The Lévy flier on a bounded domain

4. Final remarks and conclusions

Acknowledgments .................................... 18

Appendix A. General construction of the Hamiltonian-like operator for a set of random diffusing particles

Appendix B. Illustrative calculation for a small system

\section{Introduction}

Lévy flights and walks have been successfully applied to model a wide variety of phenomena displaying anomalous (superdiffusive) diffusion in many fields [1-15], including particle kinetics [16, 17], distributions of output intensities in random lasers [18, 19], and random searches in animal foraging [20-23], to name a few.

Though similar in some aspects, Lévy flights and walks differ in a number of important features. Both present step lengths drawn from the family of Lévy $\alpha$-stable distributions [9], with the Lévy index in the range $\alpha \in(0,2]$ (the limit case $\alpha=2$ corresponds to the Gaussian distribution). However, while Lévy flights are Markovian processes with jump duration (generally instantaneous) independent of the length, steps in Lévy 
walks are taken with finite (generally constant) speed, thus generating spatiotemporal correlations and leading to non-Markovian temporal evolution.

Of particular relevance is the case when the Lévy process is constrained to a onedimensional finite domain (see, e.g., $[10,11,14,20,21])$. In this context the presence of boundaries (either absorbing or reflecting) hinders considerably the calculation of the probability density function $P(x, t)$ to find the Lévy flier or walker at position $x$ in time $t$, from which all quantities of interest can be determined (e.g., rms distance, survival probability, mean first passage time, etc).

Lévy flights have been most generally studied under the framework of fractional differential equations (FDEs) and related approaches [10-16, 24-39]. In the presence of boundaries, the nonlocal character of the fractional spatial derivative of order $\alpha$ introduces a number of technical difficulties and subtleties. For instance, the non-negligible probability of long jumps, whose extension could in principle overcome the boundary sites [40], leads to the failure [41] of the method of images to determine $P(x, t)$ for $0<\alpha<2$. In particular, in the case of a single boundary this method yielded [41] a survival probability $S(t)$ with long-term behavior dependent on the Lévy index $\alpha$, at variance with the $\alpha$-independent result $S(t) \sim t^{-1 / 2}$ of the Sparre-Andersen theorem in the semi-infinite space $[42,43]$. In contrast, we remark that the method of images has been successfully employed in the diffusive (Brownian) limit with $\alpha=2[10,11,44]$. Moreover, the use of the Wiener-Hopf technique [41, 44] and the method of finite differences applied to the FDEs are also hampered due to the bounded domain $[45,46]$. We further mention the discretization of the Riesz fractional differential operator [28, $30,33,34]$, which provided the correct asymptotic behavior of the survival probability, $S(t) \sim t^{-1 / 2}$, for all $\alpha$. In this case, however, the calculation of the eigenfunctions in the expansion of $P(x, t)$ has faced [28,33-35] difficulties in the superdiffusive range of interest, $0<\alpha<2$.

More recently, two of us have applied [47] the Wiener-Hopf decomposition to the master equation of a Lévy flier between two absorbing boundaries. This approach has led to an interesting dynamical crossover of the long-term asymptotic survival probability from the power-law behavior, when only one boundary site is accessed, to the exponential time decay, when absorption takes place in both extremes. Nevertheless, in this case the calculation of $P(x, t)$ for the Lévy flier in any $t$ was not possible as well, with higher difficulties found in the short-term regime.

In this work we approach the problem of a Lévy random flier in a onedimensional bounded domain through an exact representation of its master equation as a Schrödinger-like equation analyzed in Fock space. This formalism was first proposed in $[48,49]$ in the context of classical diffusion-controlled reactions in liquid media and chemical reaction processes, and was later extended to treat other systems [50-53] (e.g., general stochastic reaction-diffusion processes and spins chains [54, 55], nonequilibrium interacting particles trapped at absorbing states [56-59], birth-death processes on a lattice [60], and gene expression [61]). More recently, one of us has also applied this method to study [62] the kinetics of more complex chemical reactions including arbitrary substrates and enzymes.

In this formalism $P(x, t)$ plays a role similar to that of the wave function in quantum mechanics. The classical Hamiltonian-like matrix is written on a basis of discrete Fock 
states representing the occupation number of every available site in the discretized finite domain, being suitably built from the matrix of jump probabilities of the Lévy flier. Here we calculate the Hamiltonian matrix for any Lévy index $\alpha \in(0,2]$. In order to obtain $P(x, t)$ for the system sizes studied here, the eingevalues and eigenvectors of the Hamiltonian matrix $\left(\sim 10^{4}\right.$ elements) are determined using a symbolic computing software. The distribution $P(x, t)$ is then computed in the form of a matrix product involving the matrix representation of the system's time evolution operator and its vector state at different times. Our results agree nicely with direct Monte Carlo simulations of a bounded particle with jump lengths drawn from a Lévy distribution in the superdiffusive range $0<\alpha<2$, averaged over a large number of runs. Good agreement is also found with results from the method of images in the diffusive limit $(\alpha=2)$ [10, 11]. Moreover, from $P(x, t)$ we also calculate the survival probability $S(t)$ for any time $t$. Both power-law and exponential long-term behaviors of $S(t)$ are obtained, and agree with the numerical simulations. In particular, the exponential decay rate of $S(t)$ nicely agrees with results from discretized Riesz fractional differential operator [28] and Wiener-Hopf [47] approximate techniques, which, in contrast, cannot provide the short-term behavior of $S(t)$. Comparisons with some exact expressions for Lévy flights in the continuous space limit also display good agreement.

This article is organized as follows. In section 2 we review the formalism of the Fock space approach applied to random particles. Results and discussions on the problem of the Lévy flier in a finite domain are presented in section 3, with concluding remarks left for section 4. Two appendices are also included to describe the construction of the Hamiltonian-like matrix of particles diffusing in a bounded domain and to provide an illustrative example of the matrix calculations in a simpler small system.

\section{Fock space formalism for bounded random particles}

We start by defining a one-dimensional finite domain, $0 \leqslant x \leqslant L$, with absorbing sites at the boundaries $x=0$ and $x=L$. Thus, when a random particle reaches $x=0$ or $x=L$ it gets absorbed and its dynamics ceases.

Consider initially the case of many identical random fliers in this interval. If they are allowed to step only at discrete positions $x_{j}=j \Delta x$, with $j=0,1, \ldots, N(=L / \Delta x)$, and no restriction exists for the simultaneous occupation of a site, then the state of the system at a given time $t$ can be represented by the set $\left\{n_{j}\right\}$ of occupation numbers of particles at each available site, with $n_{j}=0,1,2, \ldots, N_{p}$, and $N_{p}=\sum_{j} n_{j}$ as the total number of particles. In a notation similar to that of discrete Fock states in a quantum mechanical description of a system in Fock space, the state of the system of classical random particles in a discretized space can be denoted by $\left|n_{0}, n_{1}, \ldots, n_{j}, \ldots, n_{N}\right\rangle$.

In the present case of interest with a single particle, one has only $n_{j}=0,1$, and the states assume a simpler form $\{|j\rangle\}=\left\{\left|0,0, \ldots, 0, n_{j}=1,0, \ldots 0,0\right\rangle\right\}$. By denoting $P\left(x_{j}, t\right) \Delta x \equiv P(j, t) \Delta x$ as the probability to find the particle at position $x_{j}=j \Delta x$ in time $t$, or equivalently the probability that the system occupies the state $|j\rangle$ in $t$, then its statistical description at this time can be expressed by the superposition state (with $\Delta x \equiv 1)$ 


$$
|\psi(t)\rangle=\sum_{j} P(j, t)|j\rangle
$$

On the other hand, the master equation of a random particle in a one-dimensional discrete bounded space reads [44]

$$
\frac{\partial P(x, t)}{\partial t}=\sum_{x^{\prime}}\left[T\left(x, x^{\prime}\right) P\left(x^{\prime}, t\right)-T\left(x^{\prime}, x\right) P(x, t)\right] .
$$

where $x$ and $x^{\prime}$ are defined in the finite interval and $T\left(x, x^{\prime}\right)$ denotes the transition rate for the flier to perform a jump from position $x^{\prime}$ to $x$ with length $\left|x-x^{\prime}\right|$. Now, by combining the Fock space construction defined above with equation (2), the latter can be exactly represented [48-52] in the form of a real-valued Schrödinger-like equation,

$$
\frac{\partial}{\partial t}|\psi(t)\rangle=-H\left(\left\{a_{j}^{\dagger}, a_{j}\right\}\right)|\psi(t)\rangle
$$

in which the raising and lowering operators, respectively $\left\{a_{j}^{\dagger}\right\}$ and $\left\{a_{j}\right\}$, promote the creation and destruction of a random particle at position $x_{j}$. Their algebra is defined [48-52] in terms of the generic state $|k\rangle$ comprising many particles as

$$
\begin{aligned}
& a_{j}^{\dagger}|k\rangle=\left|n_{0}, n_{1}, \ldots, n_{k}, \ldots, n_{j}+1, \ldots, n_{N}\right\rangle, \\
& a_{j}|k\rangle=n_{j}\left|n_{0}, n_{1}, \ldots, n_{k}, \ldots, n_{j}-1, \ldots, n_{N}\right\rangle,
\end{aligned}
$$

with commutation relation $\left[a_{j}, a_{k}^{\dagger}\right]=\delta_{j k}$, where $\delta_{j k}$ is the Kronecker delta. As mentioned, here we are interested in the single particle case. We note in passing that the action of $a_{j}^{\dagger}$ and $a_{j}$ in the context of stochastic processes with classical particles differ from that of their quantum mechanical analogues [63], which involves factors $\sqrt{n_{j}+1}$ and $\sqrt{n_{j}}$, respectively. This is so to accommodate [48-52] the fact that the classical transition rates are proportional to the number of particles. Indeed, by applying the annihilation operator $a_{j}$ to a state $\left|n_{0}, \ldots, n_{j}, \ldots, n_{N}\right\rangle$ describing classical random flier particles at lattice positions $(0, \ldots, j, \ldots, N)$, one has $n_{j}$ ways to destroy one of the particles at position $j$, and so the $n_{j}$ prefactor arises in the second line of equation (4) [64]. Conversely, by applying the creation operator $a_{j}^{\dagger}$ to the same state, there is only one way to add an extra particle at position $j$, and then the first line of equation (4) follows. The precise connection between the rhs of equation (2) and the Hamiltonian-like operator $H$ in equation (3) needs to be established case by case in order to properly reflect the transition rates $T\left(x, x^{\prime}\right)$. In the next section we shall relate $T\left(x, x^{\prime}\right)$ to the matrix $P_{j k}$ of probabilities of jumping from site $j$ to site $k$. In appendix A we illustrate the calculation of $H$ for random particles diffusing in a bounded domain.

Once the Hamiltonian $H$ is available on the basis of Fock states, a time evolution operator can be defined, $U(0, t)=\exp \left[-t H\left(\left\{a_{j}^{\dagger}, a_{j}\right\}\right)\right]$, so that the dynamics of the system from equation (3) implies

$$
|\psi(t)\rangle=U(0, t)|\psi(0)\rangle
$$


where $|\psi(0)\rangle$ denotes its initial state, i.e., $|\psi(0)\rangle=\left|j_{0}\right\rangle$ if the flier departs from the position $x_{0}=j_{0} \Delta x$. We thus find that

$$
P(j, t)=\langle j \mid \psi(t)\rangle=\langle j|U(0, t)| \psi(0)\rangle .
$$

Also, the time-dependence of the $n$th moment of the distribution $P(j, t)$ can be obtained from

$$
\left\langle j^{n}\right\rangle(t)=\sum_{j=0}^{N} j^{n} P(j, t) .
$$

In order to proceed with the calculations, it is convenient to express the Hamiltonian $H$ in a Jordan normal form $J=Q^{-1} H Q$, so that $H=Q J Q^{-1}$, where the matrices $J$ and $Q$ are built from the eigenvalues and eigenvectors of $H$ [65]. In this representation the time evolution operator can be written in matrix form as

$$
U(0, t)=\exp (-t H)=\sum_{m=0}^{\infty} \frac{(-t)^{m}}{m !}\left(Q J Q^{-1}\right)^{m}=Q \exp (-t J) Q^{-1}
$$

where $Q^{-1}$ denotes the inverse of the matrix $Q$.

In summary, we can essentially assign the Fock space approach with the three following steps:

(a) Calculating the matrix $P_{j k}$ of probabilities of jumping from site $j$ to site $k$ (see next section and appendix B).

(b) Writing the Hamiltonian matrix $H$.

(c) Performing the Jordan decomposition to obtain the time evolution operator $U$.

Once the time evolution operator $U$ is known, one can conceivably calculate any relevant quantity of the system.

\section{The Lévy flier on a bounded domain}

We now turn to the case of a Lévy flier in a finite domain with absorbing boundaries. Its steps lengths $\ell$ are taken from the family of Lévy $\alpha$-stable distributions given by the Fourier transform [9]

$$
\left.p_{\alpha}(\ell)=\frac{1}{2 \pi} \int_{-\infty}^{\infty} \mathrm{d} k \mathrm{e}^{-b|k|^{\alpha}[1-\beta} \operatorname{sgn}(k) \Phi(k)\right]-i k(\ell-\mu),
$$

where $\alpha \in(0,2]$ is the Lévy index, $\beta \in[-1,1]$ is the skewness parameter, $b>0$ is a scale factor, $\mu$ is a location parameter, $\operatorname{sgn}$ is the sign function, and $\Phi=\tan (\pi \alpha / 2)$ if $\alpha \neq 1$ and $\Phi(k)=(-2 / \pi) \log |k|$ if $\alpha=1$. Here we consider only symmetric distributions of step lengths centered at $\ell=0$, so that $\beta=0$ and $\mu=0$ in equation (9). This means that we assign jump lengths to the right or to the left with equal probabilities, $p_{\alpha}(|\ell|)=p_{\alpha}(-|\ell|)$.

Although Lévy distributions can be cast [9-11] in terms of Fox- $\mathrm{H}$ functions, whose calculation relies on complex integrals of the Mellin-Barnes type, they generally lack closed-form expressions based on elementary functions [66-68]. In this sense, the 
Cauchy $(\alpha=1)$ and the limit case of Gaussian $(\alpha=2)$ distributions constitute notable exceptions when $\beta=0$. This fact contributes to enhance the difficulty to find analytical expressions for $P(x, t)$ of the Lévy flier on a bounded interval. Moreover, due to the non-negligible probability of long jumps for $0<\alpha<2$, the Lévy flier can eventually take a step that would lead it over one of the boundaries, effectively resulting in the absorption by the corresponding extreme site. In such a case, the method of images breaks down [41] to solve the associated FDE for $P(x, t)$ in the superdiffusive regime $0<\alpha<2$. In contrast, this method is successful when applied to the diffusion equation of a bounded Brownian walker $(\alpha=2$ limit) [10,11]. In this context, the Fock space formalism may arise as a potential alternative to compute $P(x, t)$ for the bounded Lévy flier in the superdiffusive range $0<\alpha<2$, as discussed below.

By following the procedure described in appendix A, the Hamiltonian-like operator for the bounded Lévy flier with discrete positions $j(=0,1, \ldots, N)$ and absorbing boundaries at $j=0$ and $j=N$ reads

$$
H=-\sum_{j=0}^{N} \sum_{\substack{k=1 \\ k \neq j}}^{N-1} P_{k j}\left(a_{j}^{\dagger} a_{k}-a_{k}^{\dagger} a_{k}\right),
$$

where $P_{k j}$ is the probability of jumping from site $j$ to $k$, with a role similar to that of the transition rates in equation (2). The probabilities $P_{k j}$ are calculated from

$$
P_{k j}= \begin{cases}0, & k=j ; \\ \int_{(|k-j|-1) \Delta x}^{|k-j| \Delta x} p_{\alpha}(\ell) \mathrm{d} \ell, & 1 \leqslant k, j \leqslant N-1 ; \\ \int_{(j-1) \Delta x}^{\infty} p_{\alpha}(\ell) \mathrm{d} \ell, & 1 \leqslant j \leqslant N-1, \quad k=0 ; \\ \int_{(N-j-1) \Delta x}^{\infty} p_{\alpha}(\ell) \mathrm{d} \ell, & 1 \leqslant j \leqslant N-1, \quad k=N .\end{cases}
$$

The third and fourth lines in equation (11) refer, respectively, to jumps starting at site $j$ whose length is equal to or larger than the respective distances to the left and right boundaries. These flights are thus absorbed by the sites at $k=0$ and $k=N$, respectively. We should also notice that, since we are working with symmetric Lévy distributions, then $p_{\alpha}(|\ell|)=p_{\alpha}(-|\ell|)$ and the integral in the third line above, which is in principle taken over a negative argument, from $-\infty$ to $-(j-1) \Delta x$, is in fact equivalent to the corresponding integral calculated over the positive range, from $(j-1) \Delta x$ to $\infty$. The integrals in equation (11) can be expressed for any $\alpha \in(0,2]$ in terms of the following integral,

$$
I_{\alpha}(a)=\frac{1}{\pi} \int_{0}^{\infty} \frac{\sin (k a)}{k} \mathrm{e}^{-k^{\alpha}} \mathrm{d} k
$$

with $a>0$. We note in the symmetric case with absorbing boundary conditions that $P_{k j}=P_{j k}$, with $P_{k j}=0$ if $j=0$ and $j=N$, since the flier cannot leave an extreme site when absorbed. For all other $j \neq 0, N$, one has $\sum_{k} P_{k j}=1$ to give the correct 
normalization. (Under reflecting boundary conditions, one might have $P_{k j} \neq 0$ even for $j=0$ and $j=N)$.

The matrix elements of $H$ with absorbing boundaries can be obtained for any Lévy index $\alpha \in(0,2]$ on the basis of Fock states from equations (4) and (10)-(12), yielding

$$
\langle m|H| n\rangle=-\sum_{j=0}^{N} \sum_{\substack{k=1 \\ k \neq j}}^{N-1} P_{k j}\left(\delta_{k n} \delta_{m j}-\delta_{k n} \delta_{m k}\right),
$$

with $m, n=0,1, \ldots, N$, which implies $\langle m|H| n\rangle=-P_{m n}$ if $m \neq n$ and $\langle m|H| m\rangle=1$, but with $\langle m|H| n\rangle=0$ if $n=0$ or $n=N$ due to the absorbing condition at the boundary sites $j=0$ and $j=N$. For large $N$ the eigenvalues and eigenvectors of $H$ are obtained with a symbolic computing software (Mathematica). Next, the matrices $J$ and $Q$ in the Jordan decomposition are built and the time evolution operator $U(0, t)$, equation $(8)$, is determined, along with the state of the system $|\psi(t)\rangle$ and $P(j, t)$, respectively equations (5) and (6), at any given time $t$. This allows the calculation of all other relevant quantities in any $t$. (See appendix B for an illustrative example of calculation of these matrices in a small system with $N=5$ ).

We comment that all results in this work were obtained in a notebook with Intel Core i7 processor. The Mathematica code runs efficiently in a time that depends basically on the domain length $L$ and the value of $\alpha$. For example, for $\alpha=1$ and $L=100$ it runs in less than $10 \mathrm{~s}$. For $\alpha=3 / 2$ and $L=100$, it takes about 6 min. (For comparison, direct Monte Carlo numerical simulations with the same parameters and averages over $5 \times 10^{4}$ walk runs take around 3 min to finish when $\alpha=1$ and nearly 19 min when $\alpha=3 / 2$ ).

We begin the analysis by the $\alpha=2$ Gaussian limit of the Lévy distribution (bounded Brownian flier), with $\beta=\mu=0$ and $b=1 / 2$ in equation (9), so that it displays unit variance, $\sigma^{2}=2 b=1[9]$. In this case equation (12) yields

$$
I_{\alpha=2}(a)=\frac{\operatorname{erf}(a / 2)}{2}
$$

where erf denotes the error function. If, e.g., $m \neq n$, with $m \neq 0, n \neq 0, m \neq N$ and $n \neq N$, the elements of the Hamiltonian-like matrix read

$$
\langle m|H| n\rangle=\frac{1}{2}[\operatorname{erf}((|m-n|-1) / 2)-\operatorname{erf}(|m-n| / 2)]
$$

The other matrix elements are calculated from the first, third and fourth lines of equation (11) combined with equations (13) and (14).

Figure 1 shows $P(j, t)$ as a function of the position $j$ in a domain of length $N=100$, with the starting point at the middle of the interval, $j_{0}=N / 2$. Results using the Fock space approach are depicted in circles for times $t=5$ (black), 15 (blue), and 100 (red). The curves are symmetrical with respect to the center of the interval, as expected. In all cases we observe a nice agreement with the solid lines representing the exact solution from the method of images $[10,11]$, which, as mentioned, holds in the $\alpha=2$ diffusive regime: 


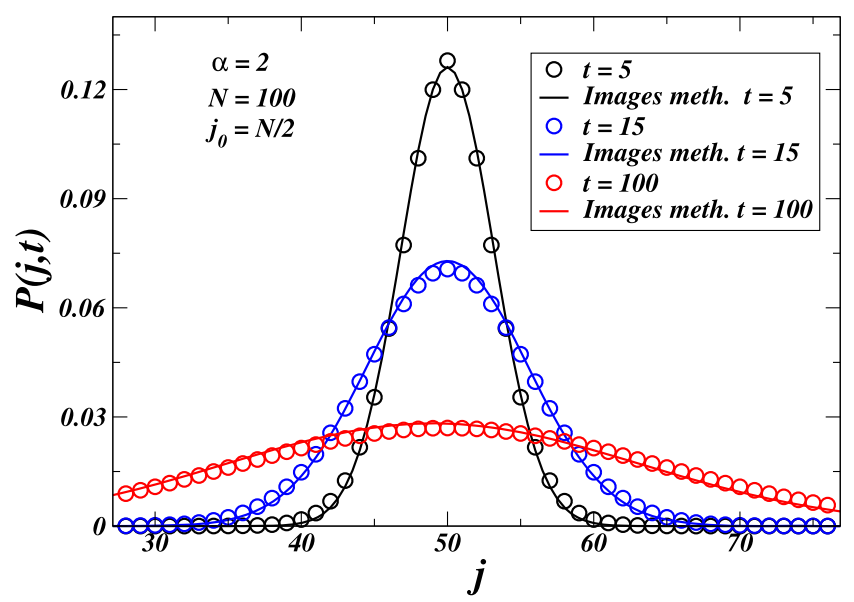

Figure 1. $P(j, t)$ for $\alpha=2$ (Gaussian limit; Brownian flier) as a function of the position $j$ in a finite domain of length $N=100$, with the starting point at the middle of the interval, $j_{0}=N / 2$. Results using the Fock space approach are depicted in circles for times $t=5$ (black), 15 (blue), and 100 (red). Fine agreement is observed with the exact solution, equation (16), from the method of images for $\alpha=2$ (solid lines).

$$
P(j, t)=\sum_{n=-\infty}^{\infty}\left[P_{0}\left(\left|j-j_{0}\right|+2 N n, t\right)-P_{0}\left(j+j_{0}+2 N n, t\right)\right],
$$

where the Gaussian function

$$
P_{0}(j, t)=\frac{1}{\sqrt{2 \pi \sigma^{2} t}} \mathrm{e}^{-j^{2} /\left(2 \sigma^{2} t\right)}
$$

is the solution of the Brownian diffusion equation in the one-dimensional unbounded space (i.e., without absorbing boundary sites). In addition, since in the $\alpha=2$ Gaussian limit the probability of long jumps is rather low, we mention that the boundary sites at $j=0$ and $j=100$ start to be reached only after $t \gtrsim 200$ (see also below the plot of the survival probability with $\alpha=2$ and $j_{0}=N / 2$ for a discussion concerning larger values of $t$ ).

We now consider a bounded Lévy flier in the superdiffusive range, with $0<\alpha<2$. In this regime with $\alpha \neq 2$, due to the failure of the method of images and further difficulties related to other analytical techniques, no exact expression for $P(x, t)$ is available. Thus, our results using the Fock space formalism are compared below with direct Monte Carlo simulations, in which a random particle is allowed to evolve in a bounded space with jump lengths drawn from the Lévy distribution $p_{\alpha}(\ell)$, with averages taken over a large number of runs. At this point, we comment that some care is in order when trying to compare the continuous time results of the Fock space approach with the discrete time results from numerical simulations. Indeed, in numerical simulations jumps take place in discrete time units $\delta t$, so that $t=n \delta t$ with $n$ denoting the number of jumps. Hence, in order to establish a proper comparison with the results from numerical simulations for very short times, $t \lesssim 10$, we have employed in the Fock space formalism the discretization scheme for small times $t$ that is usually considered for the discrete time propagator in 

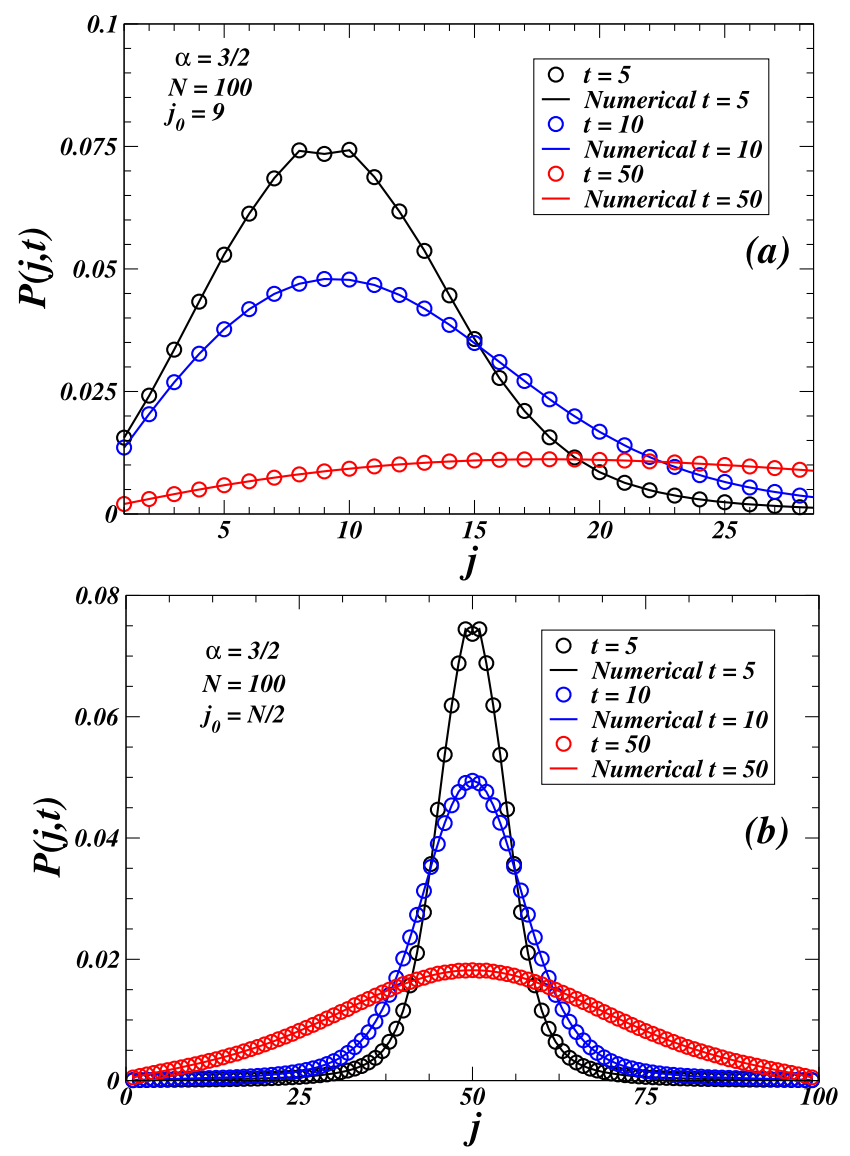

Figure 2. $P(j, t)$ for $\alpha=3 / 2$ as a function of the position $j$ in a finite domain of length $N=100$, with the starting point (a) at $j_{0}=9$ and (b) at the middle of the interval, $j_{0}=N / 2$. Results using the Fock space approach are depicted in circles for times $t=5$ (black), 15 (blue), and 50 (red). No exact solution for $P(j, t)$ is available for comparison when $\alpha \neq 2$. Nice agreement is noted with direct Monte Carlo simulations (solid lines).

quantum mechanics [63], namely, $U(0, t)=[U(\delta t)]^{n}$, with $U(\delta t)=\mathcal{I}-H \delta t$ and $\mathcal{I}$ as the identity matrix (operator). However, we point that, as the discreteness of $t$ in the numerical simulations loses relevance for larger times, $t \gtrsim 10$, then the results using both the continuous exponential and discretized forms of $U$ become indistinguishable for such values of $t$, as expected.

We first choose the value $\alpha=3 / 2$ in the superdiffusive range. (Hereafter we fix the scale factor $b=1$ in the Lévy distribution (9), unless otherwise indicated.) In this case equation (12) implies

$I_{\alpha=3 / 2}(a)=\frac{1}{\pi}\left[a \Gamma(5 / 3)_{3} F_{4}\left(\mathbf{a}_{1} ; \mathbf{b}_{1} ; c\right)-\frac{a^{3}}{9} F_{5}\left(\mathbf{a}_{2} ; \mathbf{b}_{2} ; c\right)+\frac{7 a^{5}}{405} \Gamma(4 / 3)_{3} F_{4}\left(\mathbf{a}_{3} ; \mathbf{b}_{3} ; c\right)\right]$,

where $\Gamma$ and ${ }_{p} F_{q}$ denote the gamma and generalized hypergeometric functions, respectively, with parameters $\mathbf{a}_{1}=(1 / 6,5 / 12,11 / 12), \mathbf{a}_{2}=(1 / 2,3 / 4,1,5 / 4)$, 


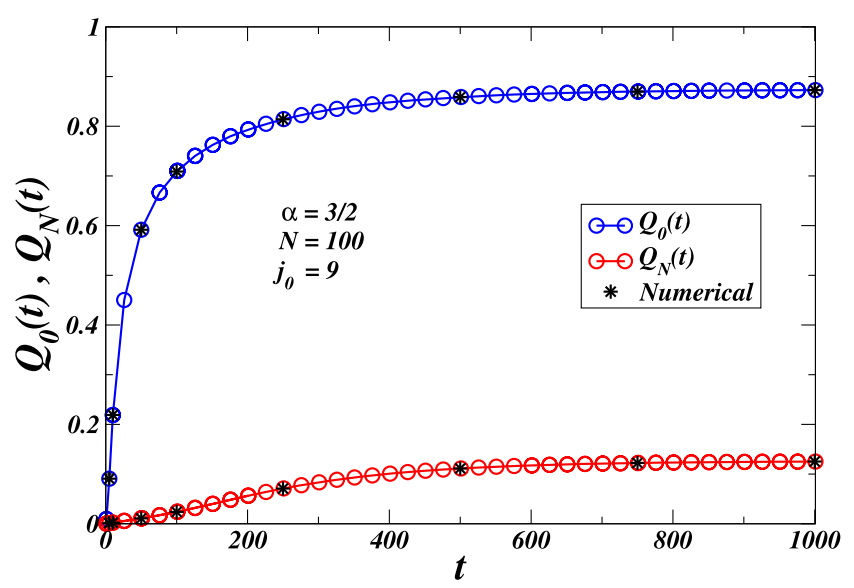

Figure 3. Probabilities $Q_{0}(t)$ and $Q_{N}(t)$ of being absorbed respectively by the boundary sites at $j=0$ and $j=N$ as a function of time $t$ for $\alpha=3 / 2$ in a finite domain of length $N=100$, with the starting point at $j_{0}=9$. Nice agreement is noted between the Fock space results (circles) and Monte Carlo simulations (stars).

$\mathbf{a}_{3}=(5 / 6,13 / 12,19 / 12), \mathbf{b}_{1}=(1 / 3,1 / 2,5 / 6,7 / 6), \mathbf{b}_{2}=(2 / 3,5 / 6,7 / 6,4 / 3,3 / 2), \mathbf{b}_{3}=$ $(7 / 6,3 / 2,5 / 3,11 / 6), c=-4 a^{6} / 729$. The off-diagonal Hamiltonian matrix elements are

$$
\langle m|H| n\rangle=I_{\alpha=3 / 2}(|m-n|-1)-I_{\alpha=3 / 2}(|m-n|),
$$

if $m \neq n$, with $m \neq 0, n \neq 0, m \neq N$ and $n \neq N$. The first, third and fourth lines of equation (11) combined with equations (13) and (18) provide the other matrix elements.

Figure 2 shows in circles the results from the Fock space approach with $N=100$ and $t=5,10,50$, for the Lévy flier starting close to the left boundary $\left(j_{0}=9\right.$, figure $\left.2(\mathrm{a})\right)$ and from the middle of the interval $\left(j_{0}=N / 2\right.$, figure 2(b)). For comparison, results from direct Monte Carlo simulations averaged over $5 \times 10^{4}$ random walk runs are depicted in solid lines. As compared to the Gaussian case $\alpha=2$, jump lengths are considerably larger when $0<\alpha<2$ (in fact, the mean jump length is infinite for $\alpha \leqslant 1$ in unbounded space). As a consequence, in this superdiffusive regime the boundary absorbing sites are reached by the flier in fewer jumps. Accordingly, figure 3 shows the time evolution of the probability of the Lévy flier being absorbed by the site at $j=0, Q_{0}(t)$, or by the site at $j=N, Q_{N}(t)$, in good agreement with the numerical simulations. These results also impact the survival probability function, as discussed below.

We now compare $P(x, t)$ in figure 4 for other values of $\alpha$ with fixed time $t$. Results shown are for $\alpha=1 / 2$ (red), 1 (blue), and 3/2 (black), with $t=5, N=100$, and $j_{0}=9$ (figure $4(\mathrm{a})$ ) and $j_{0}=N / 2$ (figure $4(\mathrm{~b})$ ). In particular, the expressions for the offdiagonal Hamiltonian matrix elements for $\alpha=1 / 2$ and $\alpha=1$ (Cauchy flier) are given, respectively, in terms of Fresnel integrals and the arctan function (see also appendix B). For all $\alpha$ a fine comparison is observed between the Fock space and Monte Carlo simulation results.

The probabilities $Q_{0}(t)$ and $Q_{N}(t)$ of being respectively absorbed by the boundary sites at $j=0$ or $j=N$ are intrinsically related to the survival probability $S(t)$, one of the most relevant quantities in bounded random walk problems [69-71], given for 

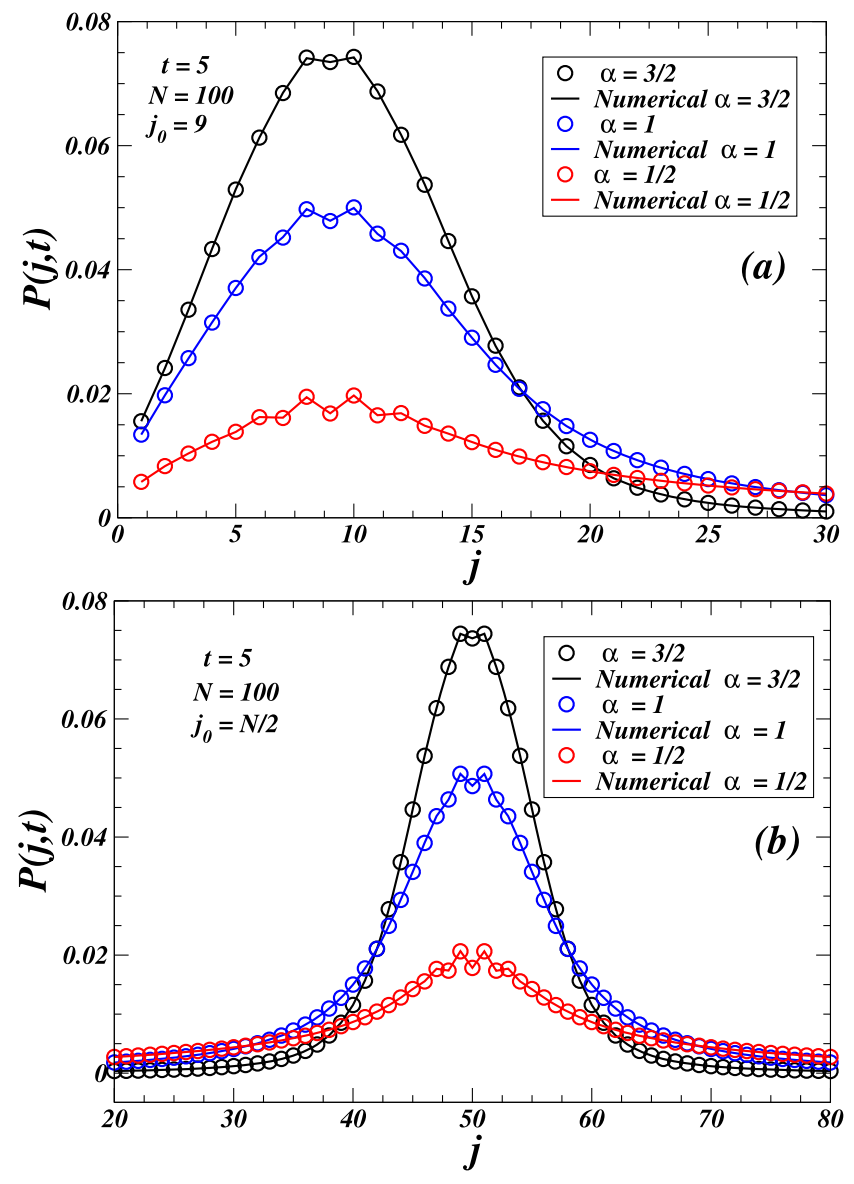

Figure 4. $P(j, t)$ for fixed time $t=5$ and $\alpha=1 / 2$ (red), 1 (blue), and $3 / 2$ (black), as a function of the position $j$ in a finite domain of length $N=100$, with the starting point (a) at $j_{0}=9$ and (b) at the middle of the interval, $j_{0}=N / 2$. Results using the Fock space approach are depicted in circles, whereas Monte Carlo simulations are shown in solid lines.

$\Delta x \equiv 1$ by

$$
S(t)=\sum_{j=1}^{N-1} P(j, t)=1-\left[Q_{0}(t)+Q_{N}(t)\right] .
$$

In figure 5 we show in circles the Fock space results of $S(t)$ in $\log -\log$ scale as a function of $t$ for the $\alpha=2$ Brownian flier with $b=1 / 2$ in a larger domain with $N=300$ and starting point $j_{0}=9$ close to the left boundary. Results from numerical simulations are depicted in solid line. In this case, since long jumps are quite rare when $\alpha=2$ the faraway right boundary at $j=300$ is not effectively reached. In practice, this situation resembles that of a random walker in a semi-infinite domain, in which the Sparre-Andersen theorem predicts $[42,43]$ the asymptotic power-law form

$$
S(t) \sim \frac{1}{\sqrt{t}}
$$




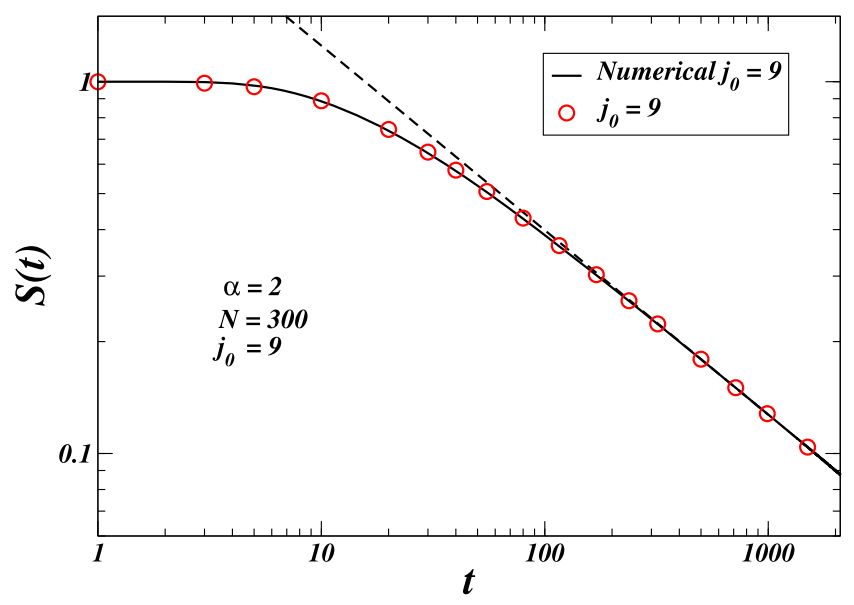

Figure 5. $\log -\log$ plot of the survival probability $S(t)$ as a function of time $t$ for the $\alpha=2$ Gaussian flier in a large extension domain with $N=300$ and starting point $j_{0}=9$ close to the left boundary. Circles and solid line depict, respectively, the results from the Fock space approach and numerical simulations. In this case only the left boundary is effectively reached by the flier for the values of $t$ shown. The long-term behavior is given by the power law $S(t) \sim t^{-\gamma}$ (dashed line), with the best-fit value $\gamma=0.491$.

Although in the present case the Lévy flier actually evolves in a finite domain, so that the conditions for the Sparre-Andersen theorem are not strictly observed, the long-term regime of the survival probability in figure 5 (dashed line) is given by $S(t) \sim t^{-\gamma}$, with the best-fit value $\gamma=0.491$. Consistently, the probability of being absorbed by the faraway boundary at $j=300$ is rather low, $8.3 \times 10^{-6}$ for $t=2000$ and $5.4 \times 10^{-10}$ for $t=1000$.

A quite interesting dynamical crossover takes place when the other boundary site also becomes progressively reached. In this case, the power-law behavior observed in figure 5 is smoothly replaced by an exponentially decaying survival probability [47, 71],

$$
S(t) \sim \mathrm{e}^{-\lambda t}
$$

In order to capture this change with an $\alpha=2$ Gaussian flier $(b=1 / 2)$, we considered in figure 6(a) a narrower interval (in comparison with figure 5), so that the right boundary site could also be possibly reached in feasible computational times. Indeed, figure 6(a) displays the Fock space (circles) and numerical simulation (solid lines) results in the linear-log plot of $S(t)$ as a function of $t$, with $N=100$ and starting points $j_{0}=9$ (black) and $j_{0}=N / 2$ (red). We observe in this case that the power-law decay becomes restricted to the low- $t$ regime, with the exponential decay of $S(t)$ emerging for larger times (dashed lines).

A similar behavior also occurs for the Cauchy flier with $\alpha=1$, as shown in figure 6(b) with same parameters as in figure 6(a). In this case, however, since jumps are much larger than when $\alpha=2$, the crossover from the power-law to the exponential behavior of $S(t)$ takes place much earlier. In particular, in equation (22) our best-fit decay rate $\lambda=0.0239$ for $\alpha=1, b=1$ and $N=100$ was found to be in excellent agreement with the results for the same parameters from both discretized Riesz fractional differential operator [28] 

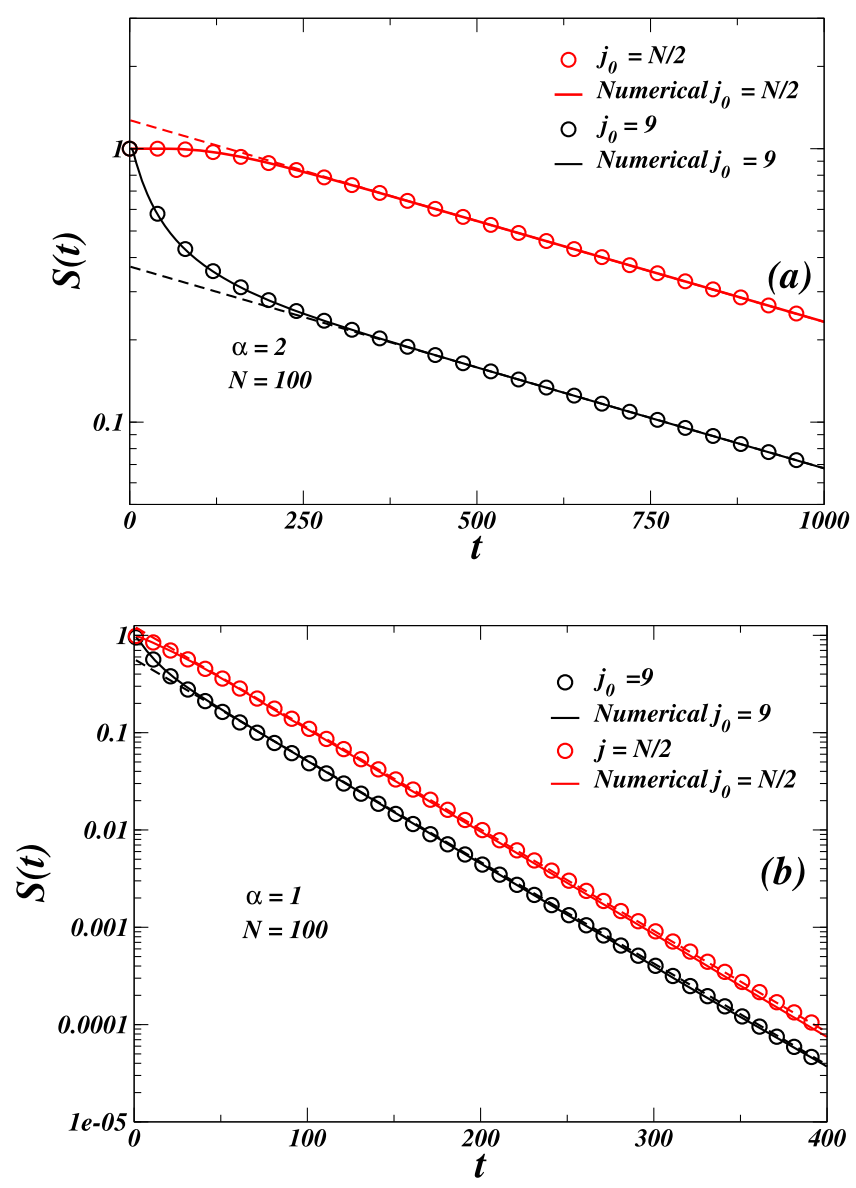

Figure 6. Linear-log plot of the survival probability $S(t)$ as a function of time $t$ for the (a) $\alpha=2$ Gaussian and (b) $\alpha=1$ Cauchy fliers in a narrower domain with $N=100$ (in comparison with $N=300$ in figure 5 ). Starting points are $j_{0}=9$ (black) and $j_{0}=N / 2$ (red). Circles and solid lines depict, respectively, the results from the Fock space approach and numerical simulations. Dashed lines are fits to the asymptotic exponential decay behavior, $S(t) \sim \mathrm{e}^{-\lambda t}$, observed when absorption takes place in both boundary sites. This contrasts with the asymptotic power-law behavior in figure 5 when only a single absorbing site (the initially closer one) is reached.

$(\lambda=0.0236)$ and Wiener-Hopf decomposition $[47](\lambda=0.0231)$ techniques (see table 1$)$. We stress, however, that these methods were not able to provide the short-term behavior of $S(t)$, which was possible in our approach due to the calculation of $P(x, t)$ for all $t$.

This similarity of results for $\lambda$ obtained through different methods might indicate the existence of some interesting connection between the Fock space formalism and the spectrum of eigenvalues $\lambda_{k}$ of the fractional Laplacian operator [28, 72, 73]. As stated in [28], the exact values of $\lambda_{k}$ are not known and their calculation still remains an open question. As a consequence, the investigation of several associated quantities is hindered by this fact. For instance, the survival probability can be cast [28] in the form $S(t)=\sum_{k} a_{k} \mathrm{e}^{-\left|\lambda_{k}\right| t}$, so that the smallest eigenvalue $\left|\lambda_{k=1}\right|$ (in absolute value) essentially 
Table 1. Decay rate $\lambda$ of the survival probability $S(t)$, equation (22), obtained from the present Fock space approach, discretized Riesz fractional differential operator method [28], and Wiener-Hopf decomposition technique [47].

\begin{tabular}{llcc}
\hline$\alpha$ & This work & Reference $[28]$ & Reference $[47]$ \\
\hline 1 & 0.0239 & 0.0236 & 0.0231 \\
2 & 0.001085 & 0.000990 & 0.000955 \\
\hline
\end{tabular}

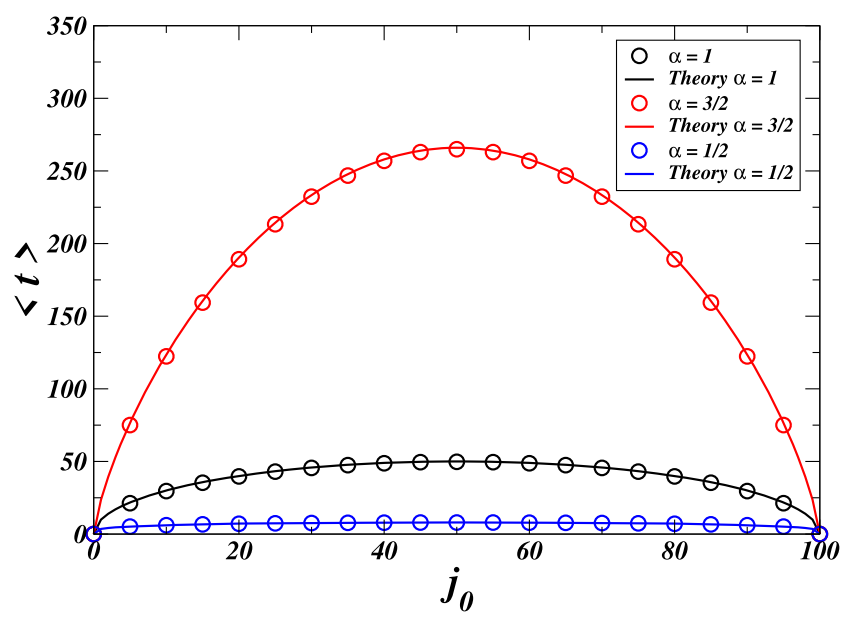

Figure 7. Comparison between the Fock space results (circles) and the exact mean first passage time, equation (24) (solid lines), of Lévy flights with $\alpha=1,1 / 2,3 / 2$, in a bounded domain of length $L=100$ as a function of the initial position $j_{0}$.

determines the long-term behavior of $S(t)$, i.e., $\lambda=\left|\lambda_{k=1}\right|$. Analogously, we generally find in the Fock space approach that $S(t)=\sum_{i} c_{i} \mathrm{e}^{-\mu_{i} t}$, with $c_{i}$ and $\mu_{i}>0$ determined from the calculation of the eigenvalues and eigenvectors of the matrix $\exp (-t H)$. Therefore, this similarity suggests that there can be some relation between the spectrum of eigenstates $\lambda_{k}$ of the fractional Laplacian operator and the eigenvalue spectrum of the Hamiltonianlike operator $H$. Indeed, besides the $\alpha=1$ Cauchy case, we also notice for the $\alpha=2$ Gaussian regime with $N=100$ that our best-fit value $\lambda=0.001085$ compares nicely with the results $\lambda=0.000990$ from [28] and $\lambda=0.000955$ from [47] (see table 1). This point deserves a deeper investigation in a future work.

One last issue regards the approach to the continuous space limit by considering $\Delta x / L \rightarrow 0$ in the present discrete space formalism. For instance, the mean first passage time has the exact result for Lévy flights in the bounded continuous space [33, 34, 74],

$$
\langle t\rangle\left(x_{0}\right)=\frac{1}{\Gamma(1+\alpha)}\left[x_{0}\left(L-x_{0}\right)\right]^{\alpha / 2} .
$$

We notice that the convergence rate of the Fock space results to equation (23) depends on both $\alpha$ and $\Delta x / L$. For example, the maximum relative error with respect to the exact 


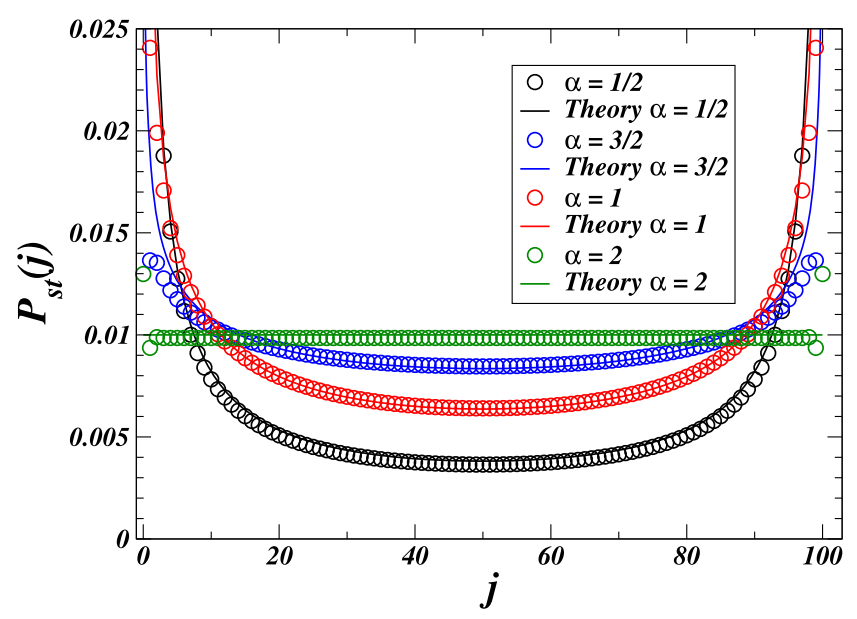

Figure 8. Comparison between the Fock space results (circles) and the exact stationary limit of $P(x, t)$, equation (25) (solid lines), of Lévy flights with $\alpha=$ $1,1 / 2,3 / 2,2$, as a function of the position $j$ in a bounded domain of length $L=100$, with $j_{0}=L / 2$ and reflecting boundaries.

expression (i.e., the relative error with the starting point in the middle of the interval, $x_{0}=L / 2$ ) decreases when $\alpha=1$ from 0.032 for $\Delta x / L=0.01$ to 0.011 for $\Delta x / L=0.002$, while when $\alpha=1 / 2$ it decreases from 0.062 for $\Delta x / L=0.01$ to 0.032 for $\Delta x / L=0.002$. Smaller $\Delta x / L$ progressively approach the exact result in continuous space, as expected. We also observe that the convergence takes place much faster (maximum relative error of 0.004 for $\Delta x / L=0.01$ with $\alpha=1 / 2)$ when $P_{k j}$ in equation (11) is given by the continuous space approximation $[28] P_{k j}=-A(|k-j|) / A(0)$ with

$$
A(n)=\frac{\Gamma(-\alpha / 2+n) \Gamma(\alpha+1)}{\pi \Gamma(1+\alpha / 2+n)} \sin (\pi \alpha / 2),
$$

for $1 \leqslant k, j \leqslant N-1 ; P_{k j}$ for the jumps absorbed by the boundaries at $k=0$ and $k=N$ is calculated by summing (24) over the jump lengths that exceed the distance to the respective extreme sites. We note, however, that equation (11) in the limit $\Delta x / L \rightarrow 0$ is quite general and applies to any distribution $p(\ell)$ of step lengths, while equation (24) is specific of Lévy flights. The results shown in figure 7 display nice agreement between equation (23) (solid lines) and the Fock space approach (circles).

We can also apply the Fock space formalism to treat the case with reflecting boundaries. In this context, the stationary regime of $P(x, t)$ in the continuous space domain $x \in[0, L]$ and initial position $x_{0}=L / 2$ is exactly given by [75]

$$
P_{s t}(x)=\frac{L^{1-\alpha}[x(L-x)]^{\alpha / 2-1} \Gamma(\alpha)}{[\Gamma(\alpha / 2)]^{2}} .
$$

We observe in figure 8 that equation (25) (solid lines) and the Fock space results (circles) agree well for a number of values of $\alpha$. Eventually we note that our Fock space results cannot reach the singularities of $P_{s t}(x)$ at the boundaries, even though the agreement apart from the extremes is good. 


\section{Final remarks and conclusions}

In this work we have revisited the problem of computing the probability distribution $P(x, t)$ and survival probability $S(t)$ of a Lévy random flier on a bounded domain. For so, we have proposed a different approach based on a representation of its master equation as a Schrödinger-like equation in Fock space.

Due to the existence of long jumps and superdiffusive dynamics with the Lévy index in the range $0<\alpha<2$, the calculation of $P(x, t)$ and $S(t)$ for the bounded Lévy flier is considerably hindered. The current methods either give asymptotic results or fail to provide the long-term behavior of observables in the superdiffusive regime. In the Fock space approach, the Hamiltonian-like matrix can be calculated for any value of the Lévy index $\alpha \in(0,2]$, and the computation of its set of eigenvalues and eigenvectors in large systems is performed using a symbolic computing software. Though the Fock space approach does not provide closed-form expressions for $P(x, t)$ and $S(t)$, our results for $P(x, t)$ display fine agreement with direct Monte Carlo simulations in the superdiffusive regime $0<\alpha<2$ and the method of images in the $\alpha=2$ Gaussian limit, for distinct initial positions and the entire range of times $t$.

Moreover, our calculation of the survival probability $S(t)$ also provided a nice characterization of both possible long-term asymptotic regimes (power-law and exponential), depending on whether only one or both absorbing sites are effectively accessed by the flier. We observe that the present techniques cannot yield [41] the asymptotic power-law behavior of $S(t)$ for $0<\alpha<2$, or are not able to determine $S(t)$ in the whole extension of $t$, but only in its long-term asymptotic limit. The continuous space limit of the Fock space formalism is also in good agreement with some exact results of Lévy flights on bounded domains.

We remark that the Fock space formalism is general enough to be readily extended to other types of random particles with arbitrary distributions of jump lengths $p(\ell)$, including biased and/or asymmetric ones, different boundary conditions, and even ensembles of several particles diffusing simultaneously on a bounded domain. Indeed, we emphasize that while some methods are particularly associated with specific forms of jump length distributions $p(\ell)$ (e.g., the discretization of the fractional Laplacian operator related to the Lévy jump length distribution [28]), the Fock space approach can be promptly applied to any $p(\ell)$ inserted in equation (11). In particular, in recent years there has been a growing interest, in contexts as diverse as random lasers and random search foraging, related to less conventional forms of $p(\ell)$, such as hyper-exponentials, attenuated power laws, or multiple-scale expressions [19, 20, 76].

We can mention as well a more methodological gain associated with the Fock space approach. In principle one could argue that it is also possible to generate a matrix representation of the master equation (2) symbolically by using the transition rates in equation (11) for a given finite basis, and this procedure would also conceivably lead to results analogous to ours regardless the Fock space representation. However, the mapping between the stochastic and quantum mechanics using the Fock space representation for Lévy flights has also further striking conceptual and methodological consequences [77]. It not only entails the access to numerous insights and computational techniques 
for generating and studying the matrix representation of Lévy flights as a Hamiltonianlike operator and the time evolution operator $\exp (-t H)$. It also provides the prospect of using the powerful set of tools from quantum mechanics for a better understanding of Lévy flights. At this point, we comment that, although the Fock space approach does not deal directly with the flier's single trajectories, just like in quantum mechanics, it provides $P(x, t)$ and from this information one can calculate all relevant quantities. Also, by relating the master equation for Lévy flights to Feynman path integrals one may allow effective perturbation methods to be employed. In another example, time-dependent quantum perturbation theory has been already applied to estimate the survival probability in lattice models with an absorbing state [56, 57], and could be employed as well in the context of Lévy processes. Furthermore, one could also map master equations for different formulations of Lévy flights onto exactly solvable statistical mechanics models [78], such as the Ising model or the Potts model, which may generate further analytical insights in the field.

From the point of view of applications, the Fock space approach employed to the calculation of $P(x, t)$ can help to improve, for example, the understanding of interfacial molecular searches using efficient Lévy foraging dynamics, as recently pointed in the case of the search process of DNA molecules for complementary DNA [79].

We thus hope that our work can contribute to enhance the understanding and improve the set of tools available for the statistical physics approach of general bounded random walks and flights.

\section{Acknowledgments}

We thank Thiago A Sobral for fruitful discussions in the early stages of this work. EPR also thank F Bartumeus and the hospitality of the Spanish institutions. This work was supported by CNPq, CAPES, and FACEPE through the Pronex project APQ-06021.05/14 (Brazilian agencies).

\section{Appendix A. General construction of the Hamiltonian-like operator for a set of random diffusing particles}

Here we discuss, in general terms, the derivation of the Hamiltonian-like operator $H$, defined in equation (3), for a system of classical non-interacting identical particles diffusing randomly on a one-dimensional finite domain of discrete sites $j=0,1, \ldots, N$.

We consider that the number of particles at a given site $j$ is $n_{j}=0,1,2, \ldots, N_{p}$, with the total number of particles constrained to $N_{p}=\sum_{j} n_{j}$. We also define the vector $\mathbf{n} \equiv\left(n_{1}, n_{2}, \ldots, n_{N}\right)$ and, whenever convenient, we use below the notation $\mathbf{n}^{(k, j)}$ $=\left(n_{1}, n_{2}, \ldots, n_{k-1}, n_{k+1}, \ldots, n_{j-1}, n_{j+1}, \ldots, n_{N}\right)$, so that $\mathbf{n}=\left(\mathbf{n}^{(k, j)}, n_{k}, n_{j}\right)$.

The quantity $P(\mathbf{n}, t)$ is the probability density for a given set $\mathbf{n}$ of occupation numbers of particles in time $t$. Single-particle transitions can be represented in the master 
equation [50-53],

$$
\frac{\partial P(\mathbf{n}, t)}{\partial t}=\sum_{j, k} D_{k j}\left[\left(n_{k}+1\right) P\left(\mathbf{n}^{(k, j)}, n_{k}+1, n_{j}-1, t\right)-n_{k} P(\mathbf{n}, t)\right],
$$

in which the transition rate for a particle to jump from site $j$ to site $k$ is proportional to the number of particles, with proportionality constant $D_{k j}=D_{j k}$ (symmetrical jumps) and $D_{j j}=0$. (Simultaneous multiple-particle transitions occur with vanishing probability.) In a representation of Fock states $\{|\mathbf{n}\rangle\}$, the state of the system in time $t$ reads

$$
|\psi(t)\rangle=\sum_{\mathbf{n}} P(\mathbf{n}, t)|\mathbf{n}\rangle
$$

where each set $\mathbf{n}$ in the sum above has total number of particles $N_{p}$, so that

$$
\frac{\partial}{\partial t}|\psi(t)\rangle=\sum_{\mathbf{n}} \sum_{j, k} D_{k j}\left[\left(n_{k}+1\right) P\left(\mathbf{n}^{(k, j)}, n_{k}+1, n_{j}-1, t\right)-n_{k} P(\mathbf{n}, t)\right]|\mathbf{n}\rangle .
$$

Now, by using equation (4) we write

$$
a_{j}^{\dagger} a_{k}\left|\mathbf{n}^{(k, j)}, n_{k}+1, n_{j}-1\right\rangle=\left(n_{k}+1\right)|\mathbf{n}\rangle,
$$

which implies

$$
\begin{aligned}
\frac{\partial}{\partial t}|\psi(t)\rangle= & \sum_{\mathbf{n}} \sum_{j, k} D_{k j}\left\{a_{j}^{\dagger} a_{k} P\left(\mathbf{n}^{(k, j)}, n_{k}+1, n_{j}-1, t\right) \mid \mathbf{n}^{(k, j)}, n_{k}\right. \\
& \left.\left.+1, n_{j}-1\right\rangle-a_{k}^{\dagger} a_{k} P(\mathbf{n}, t)|\mathbf{n}\rangle\right\} \\
= & \sum_{j, k} D_{k j}\left\{a_{j}^{\dagger} a_{k} \sum_{\mathbf{n}} P\left(\mathbf{n}^{(i, j)}, n_{k}+1, n_{j}-1, t\right) \mid \mathbf{n}^{(k, j)}, n_{k}\right. \\
& \left.\left.+1, n_{j}-1\right\rangle-a_{k}^{\dagger} a_{k} \sum_{\mathbf{n}} P(\mathbf{n}, t)|\mathbf{n}\rangle\right\} \\
= & \left\{\sum_{j, k} D_{k j}\left(a_{j}^{\dagger} a_{k}-a_{k}^{\dagger} a_{k}\right)\right\}|\psi(t)\rangle .
\end{aligned}
$$

By comparing this result with equation (3), and using that $D_{k j}=D_{j k}$ and $D_{j j}=0$, we obtain the Hamiltonian-like operator,

$$
H=\sum_{j, k} D_{k j}\left(a_{j}^{\dagger} a_{k}-a_{k}^{\dagger} a_{k}\right) .
$$

We note that the first term in $H$ can be assigned to the jump of a particle from site $k$ to $j$ with probability $D_{k j}$. The number operator (second term) arises due to the algebra 
of the raising and lowering operators, and are ultimately related to the conservation of the total number of particles in a jump transition.

In the case of absorbing boundary conditions at the sites $j=0$ and $j=N$ (see text below equation (11)), with $D_{k k}=0, D_{k j}=P_{k j}$ if $j \neq 0$ and $j \neq N$, and $D_{k j}=0$ if $j=0$ and $j=N$, equation (31) becomes equation (10).

\section{Appendix B. Illustrative calculation for a small system}

We now provide an illustrative example of the calculation of the Hamiltonian-like matrix $H$, equation (13), and the associated forms $Q, J$ and $U(0, t)$ in the simple case of a small system with $N=5$ (i.e., only six sites available to the Lévy flier, $j=0,1, \ldots, N$ ).

We consider, for instance, the case of the Cauchy flier with $\alpha=1$. The integral (12) for $\alpha=1$ reads

$$
I_{\alpha=1}(a)=\frac{\arctan (a)}{\pi},
$$

from which the off-diagonal elements of the Hamiltonian matrix can be obtained as

$$
\left[H_{\alpha=1}\right]_{m n}=[\arctan (|m-n|-1)-\arctan (|m-n|)] / \pi
$$

if $m \neq n, m \neq 1, n \neq 1, m \neq 6$ and $n \neq 6$. The other matrix elements are calculated from the first, third and fourth lines of equation (11) combined with equations (13) and (32). We thus obtain the Hamiltonian matrix for $\alpha=1$,

$$
H_{\alpha=1}=\left(\begin{array}{cccccc}
0 & -1 / 2 & -1 / 4 & -0.148 & -0.102 & 0 \\
0 & 1 & -1 / 4 & -0.102 & -0.045 & 0 \\
0 & -1 / 4 & 1 & -1 / 4 & -0.102 & 0 \\
0 & -0.102 & -1 / 4 & 1 & -1 / 4 & 0 \\
0 & -0.045 & -0.102 & -1 / 4 & 1 & 0 \\
0 & -0.102 & -0.148 & -1 / 4 & -1 / 2 & 0
\end{array}\right) .
$$

We observe that the columns $n=1$ and $n=6$ are null due to the absorbing sites at the boundary positions $j=0$ and $j=N=5$, respectively. Further, we also note that the sum of each column of $H_{\alpha=1}$ is null (we show above only the first three decimal places), as a consequence of the sum to unit of the total probability for the flier to be anywhere in the bounded interval.

By using the Jordan decomposition method [65], the matrices $Q_{\alpha=1}$ and $J_{\alpha=1}$ can be built from the eigenvalues and eigenvectors of $H_{\alpha=1}$, yielding

$$
Q_{\alpha=1}=\left(\begin{array}{cccccc}
1 & 0 & 0.575 & -0.270 & 0.138 & 0.05 \\
0 & 0 & -0.247 & 0.579 & -0.554 & -0.327 \\
0 & 0 & -0.329 & 0.303 & 0.416 & 0.625 \\
0 & 0 & -0.329 & -0.303 & 0.416 & -0.625 \\
0 & 0 & -0.247 & -0.579 & -0.554 & 0.327 \\
0 & 1 & 0.575 & 0.270 & 0.138 & -0.05
\end{array}\right)
$$


and

$$
\mathrm{e}^{-t J_{\alpha=1}}=\left(\begin{array}{cccccc}
1 & 0 & 0 & 0 & 0 & 0 \\
0 & 1 & 0 & 0 & 0 & 0 \\
0 & 0 & \mathrm{e}^{-0.485 t} & 0 & 0 & 0 \\
0 & 0 & 0 & \mathrm{e}^{-0.968 t} & 0 & 0 \\
0 & 0 & 0 & 0 & \mathrm{e}^{-1.219 t} & 0 \\
0 & 0 & 0 & 0 & 0 & \mathrm{e}^{-1.327 t}
\end{array}\right)
$$

The first two columns in $Q_{\alpha=1}$ represent the eigenvectors $|1,0,0,0,0,0\rangle$ and $|0,0,0,0,0,1\rangle$, which indicate that, once the boundary site $j=0$ or $j=5$ is reached, the flier is absorbed and cannot leave this position. On the other hand, the matrix $J_{\alpha=1}$ is diagonal, with elements given by the eigenvalues of $H_{\alpha=1}$. Again, due to the presence of the boundary absorbing sites two eigenvalues are null, which correspond to the unit values in the first two columns of $\exp \left(-t J_{\alpha=1}\right)$. The other eigenvalues of $H_{\alpha=1}$ can be read off from the decay constants in the exponentials above. Finally, the evolution operator as a function of time $t$ is obtained from the matrix product $U_{\alpha=1}(0, t)=Q_{\alpha=1} \exp \left(-t J_{\alpha=1}\right) Q_{\alpha=1}^{-1}$, equation (8), where $Q_{\alpha=1}^{-1}$ denotes the inverse matrix of $Q_{\alpha=1}$.

Any relevant quantity can be calculated from the time evolution operator. For example, in the specific case with $j=j_{0}=2$ equation (6) leads to the following exponential decay,

$$
P_{\alpha=1}\left(j_{0}, t\right)=\left\langle j_{0}\left|U_{\alpha=1}(0, t)\right| j_{0}\right\rangle=0.320 \mathrm{e}^{-0.485 t}+0.180\left(\mathrm{e}^{-0.968 t}+\mathrm{e}^{-1.219 t}\right)+0.393 \mathrm{e}^{-1.327 t} .
$$

The above procedure can be applied to any other value of $\alpha \in(0,2]$ by using equations (11)-(13). In particular, for the values $\alpha=3 / 2$ and $\alpha=2$ considered in this work we use, respectively, equations (18), (19) and (14), (15) to build the Hamiltonian matrix $H$ and the associated matrices.

\section{References}

[1] Montroll E W and Shlesinger M F 1984 Nonequilibrium Phenomena II: From Stochastics to Hydrodynamics ed J Leibowitz and E W Montroll (Amsterdam: North-Holland)

[2] Havlin S and Ben-Avraham D 1987 Adv. Phys. 36695

[3] Shlesinger M F, Zaslavsky G M and Klafter J 1993 Nature 36331

[4] West B J and Deering W 1994 Phys. Rep. 2461

[5] Shlesinger M F, Zaslavsky G M and Frisch U (ed) 1995 Lévy Flights and Related Topics in Physics (Berlin: Springer)

[6] Hughes B D 1995 Random Walks and Random Environments (Oxford: Clarendon)

[7] Klafter J, Shlesinger M F and Zumofen G 1996 Phys. Today 4933

[8] Kutner R, Pekalski A and Sznajd-Weron K (ed) 1999 Anomalous Diffusion: From Basics to Applications (Berlin: Springer)

[9] Zolotarev V M and Uchaikin V M 1999 Chance and Stability: Stable Distributions and their Applications (Utrecht: VSP BV)

[10] Metzler R and Klafter J 2000 Phys. Rep. 3391

[11] Metzler R and Klafter J 2004 J. Phys. A 37 R161

[12] Chechkin A V, Gonchar V Y, Klafter J and Metzler R 2006 Adv. Chem. Phys. 133439 
[13] Metzler R, Chechkin A V, Gonchar V Y and Klafter J 2007 Chaos, Solitons Fractals 34129

[14] Klafter J and Sokolov I M 2011 First Steps in Random Walks (Oxford: Oxford University Press)

[15] Klafter J, Lim S C and Metzler R (ed) 2012 Fractional Dynamics: Recent Advances (Singapore: World Scientific)

[16] Zaburdaev V, Denisov S and Klafter J 2015 Rev. Mod. Phys. 87483

[17] Mandelbrot B B and Pignoni R 1983 The Fractal Geometry of Nature vol 173 (New York: W H Freeman and Company)

[18] Bardou F 2002 Lévy Statistics and Laser Cooling: How Rare Events Bring Atoms to Rest (Cambridge: Cambridge University Press)

[19] Lima B C, Gomes A S L, Pincheira P I R, Moura A L, Gagné M, Raposo E P, de Araújo C B and Kashyap R 2017 J. Opt. Soc. Am. B 34293

[20] Viswanathan G M, da Luz M G E, Raposo E P and Stanley H E 2011 The Physics of Foraging (Cambridge: Cambridge University Press)

[21] Méndez V, Campos D and Bartumeus F 2014 Stochastic Foundations in Movement Ecology (Berlin: Springer)

[22] Viswanathan G M, Raposo E P and da Luz M G E 2008 Phys. Life Rev. 5133

[23] Raposo E P, Buldyrev S V, da Luz M G E, Viswanathan G M and Stanley H E 2009 J. Phys. A 42434003

[24] Fogedby H C 1994 Phys. Rev. Lett. 732517

[25] Fogedby H C 1994 Phys. Rev. E 501657

[26] Jespersen S, Metzler R and Fogedby H C 1999 Phys. Rev. E 592736

[27] Podlubny I 1999 Fractional Differential Equations (London: Academic)

[28] Zoia A, Rosso A and Kardar M 2007 Phys. Rev. E 76021116

[29] Kantor Y and Kardar M 2007 Phys. Rev. E 76061121

[30] Latzav E and Adda-Bedia M 2008 Europhys. Lett. 8330006

[31] Lucena L S, da Silva L R, Evangelista L R, Lenzi M K, Rossato R and Lenzi E K 2008 Chem. Phys. 34490

[32] García-García R, Rosso A and Schehr G 2012 Phys. Rev. E 86011101

[33] Buldyrev S V, Havlin S, Kazakov A Y, da Luz M G E, Raposo E P, Stanley H E and Viswanathan G M 2001 Phys. Rev. E 64041108

[34] Buldyrev S V, Gitterman M, Havlin S, Kazakov A Y, da Luz M G E, Raposo E P, Stanley H E and Viswanathan G M 2001 Physica A 302148

[35] Gitterman M 2000 Phys. Rev. E 626065

[36] Padash A, Chechkin A V, Dybiec B, Pavlyukevich I, Shokri B and Metzler R 2019 J. Phys. A 52454004

[37] Dybiec B, Gudowska-Nowak E and Hänggi P 2006 Phys. Rev. E 73046104

[38] Dybiec B, Gudowska-Nowak E, Barkai E and Dubkov A A 2017 Phys. Rev. E 95052102

[39] Dybiec B and Sokolov I M 2015 Comput. Phys. Commun. 18729

[40] Koren T, Lomholt M A, Chechkin A V, Klafter J and Metzler R 2007 Phys. Rev. Lett. 99160602

[41] Chechkin A V, Metzler R, Gonchar V Y, Klafter J and Tanatarov L V 2003 J. Phys. A 36 L537

[42] Sparre Andersen E 1953 Math. Scand. 1263

[43] Sparre Andersen E 1954 Math. Scand. 2195

[44] Feller W 1968 An Introduction to Probability Theory and its Applications vol 2 (New York: Wiley)

[45] Ciesielski M and Leszczynski J 2006 J. Theor. Appl. Mech. 44393

[46] Krepysheva N, Di Pietro L and Néel M-C 2006 Phys. Rev. E 73021104

[47] Araújo H A and Raposo E P 2016 Phys. Rev. E 94032113

[48] Doi M 1976 J. Phys. A 91465

[49] Doi M 1976 J. Phys. A 91479

[50] Mattis D C and Glasser M L 1998 Rev. Mod. Phys. 70979

[51] Baez J C and Biamonte J D 2018 Quantum Techniques in Stochastic Mechanics (Singapore: World Scientific)

[52] Isaacson S A 2008 J. Phys. A 41065003

[53] Grassberger P and Scheunert M 1980 Fortschr. Phys. 28547

[54] Alcaraz F, Droz M, Henkel M and Rittenberg V 1994 Ann. Phys., NY 230250

[55] Henkel M, Orlandini E and Santos J 1997 Ann. Phys., NY 259163

[56] Dickman R and Jensen I 1991 Phys. Rev. Lett. 672391

[57] Jensen I and Dickman R 1993 J. Stat. Phys. 7189

[58] Dickman R 1989 J. Stat. Phys. 55997

[59] Dickman R, Wang J-S and Jensen I 1991 J. Chem. Phys. 948252

[60] Peliti L 1985 J. Phys. 461469

[61] Sasai M and Wolynes P G 2003 Proc. Natl Acad. Sci. USA 1002374

[62] Santos F A N, Gadêlha H and Gaffney E A 2015 Phys. Rev. E 92062714

[63] Sakurai J J 1994 Modern Quantum Mechanics (Reading, MA: Addison-Wesley) 
[64] Täuber U C, Howard M and Vollmayr-Lee B P 2005 J. Phys. A 38 R79

[65] Dennery A K P 1996 Mathematics for Physicists (New York: Dover)

[66] Penson K A and Górska K 2010 Phys. Rev. Lett. 105210604

[67] Górska K and Penson K A 2011 Phys. Rev. E 83061125

[68] Rocha É C, da Luz M G E, Raposo E P and Viswanathan G M 2019 Phys. Rev. E $100010103(\mathrm{R})$

[69] Cox D R and Miller H D 1965 The Theory of Stochastic Processes (New York: Wiley)

[70] Blumenthal R M, Getoor R K and Ray D B 1961 Trans. Am. Math. Soc. 99540

[71] Dybiec B, Gudowska-Nowak E and Chechkin A 2016 J. Phys. A 49504001

[72] Kwasnicki M 2012 J. Funct. Anal. 2622379

[73] Garbaczewski P and Stephanovich V 2019 Phys. Rev. E 99042126

[74] Getoor R K 1961 Trans. Am. Math. Soc. 10175

[75] Denisov S I, Horsthemke W and Hänggi P 2008 Phys. Rev. E 77061112

[76] Santana-Filho J V, Raposo E P, Macêdo A M S, Vasconcelos G L, Viswanathan G M, Bartumeus F and da Luz M G E 2020 J. Stat. Mech. 2020023406

[77] Garbaczewski P and Stephanovich V 2013 J. Math. Phys. 54072103

[78] Baxter R J 2007 Exactly Solved Models in Statistical Mechanics (New York: Dover)

[79] Monserud J H and Schwartz D K 2016 Phys. Rev. Lett. 116098303 